\title{
ISO 15189:2012 Accreditation and Certification: Are
}

\section{You Ready?}

\section{Rajendra Dev Bhatt ${ }^{1 *}$, Raj Kumar Shrestha', Dhruba Acharya ${ }^{2}$}

"The quality of shoes reflects the status of man and standard of toilets reflect the status of family": we mean to say- quality matters in dayto-day living. Despite less recognition and prioritization of clinical laboratory in former years, the recent advancement in modern medicine has compelled the health professionals to intensify its importance in diagnosis, treatment, prognosis and management of diseases. It is estimated that $70-80 \%$ of clinical decisions are based upon information derived from laboratory results [1]. The simultaneous concern on quality of clinical diagnosis has been noted in developing country like Nepal. A huge investment in automation for the sake of quality services has also justified the fact that quality definitely matters. However, the enigma of quality service in clinical laboratory still exists in our health environment. Hence, we attempted to poke a part of the process of developing quality services in diagnostic fraternity.

Although the term 'quality' is understood by everyone, the perception might differ from person to person. Tirupathi R. Chandrupatla has summarized few definition of the term quality. Crossby has defined quality as a conformance to requirements and Juran has defined it as a fitness for use. W. Edwards Deming defined quality as: 'Good quality means a predictable degree of uniformity and dependability with a quality standard suited to the customer'. According to American Society for Quality (ASQ), it denotes an excellence in goods and services, especially to the degree they conform to requirements and satisfy customers [2].

In the context of clinical laboratory service, there are few national and international bodies/systems which look after the outcomes of the quality. ISO 15189 Medical Laboratories- Requirements for Quality and Competence was developed by the International Organization for Standardization's Technical Committee 212 (ISO/TC 212). For the sake of improving the quality of laboratory services, evaluation of quality system in every step of total testing and technical compliances that requires appropriate education and training, the latest version of 15189 i.e. ISO 15189:2012 was developed $[3,4]$. So far, the standard is adopted by medical laboratories in over 200 countries [5].

However, the accreditation and certification of our clinical laboratories with ISO is still in primitive stage. We might not be aware of the fact that there are standard protocols and third party accreditation system to prove our quality service. There are mainly three types of certifications in the testing laboratories- first party, second party and third party certifications. First party certification is the one where laboratory belongs to the user, who is satisfied and uses for his own jobs. Second party certification defines that laboratory does not belong to user but user using his own means certifies the laboratory, and uses for his own requirements. Similarly, third party certification belongs to certifying/accreditation body for laboratories is neither the owner for the laboratory nor is the user [6].

In our context of laboratory services, patients get puzzled knowing test rates discrepancies among laboratories. However, the patients later on convinced that the rate differs on the basis of quality of the results. In that mean, it is of utmost importance to understand what is standard and which one is better. A standard is a document that provides requirements, specifications, guidelines or characteristics that can be used consistently to ensure that materials, products, processes and services are fit for their purpose. International Standards Organization (ISO: 15189) ensure that products and services are safe, reliable and of good quality [7].

Nevertheless, the dogmas and enigmas regarding ISO 15189 still exist in our clinical laboratory settings. So, many curious queries are still jeopardizing the health professionals and even patients. The major query among many is about the objective of ISO. Some people think that ISO is a commercial organization. However, we

${ }^{1}$ Department of Biochemistry, ${ }^{2}$ Department of Microbiology, Kathmandu University School of Medical Sciences, Kavre, Nepal

Correspondence to: Rajendra Dev Bhatt, Lecturer/Clinical Biochemist, Department of Biochemistry, Kathmandu University School of Medical Sciences, Kavre, Nepal.Email: bhattdev.rajendra@gmail.com 
believe that ISO is an independent, nongovernmental membership organization and the world's largest developer of voluntary internal standards. Had there been business, they would have been ranked among the richest people, as we know that ISO has been expanded in more than 200 countries so far. Rather, there are few benefits of ISO 15189 accreditation and certification. National and international recognition, public and industry acceptance, assurance to clients, global equivalence, reliability etc. are few of them [6].

Dev Morris has clearly justified in his blog that ISO 15189 does not replace Clinical Laboratory Improvement Amendments (CLIA) or any other quality control governing bodies. Instead ISO 15189 is expensive. However, it is also true that the return on investment can be considerable [5]. To answer the query whether a laboratory can self-declare its quality control against ISO 15189, the official site of ISO has mentioned that without an evaluation by an accreditation body of ISO 15189, no laboratory can process by itself [4].

Nevertheless, ISO accreditation and certification is not mandatory for our laboratory settings. We can produce quality service without this accreditation. However, can a laboratory selfproclaim its quality by itself? It would definitely assure more quality if the laboratory has third party accreditation and certification which systematized the entire process of producing quality results. In this regard, accreditation with ISO 15189:2012 is not a bad idea for our laboratory setting in Nepal. Although this accreditation does not guarantee hundred percent error free test results, it definitely minimizes the errors.

Conflict of Interest: None declared

\section{References}

1. Forsman RW. Why is the laboratory an afterthought for managed care organizations? Clin Chem. 1996 May;42(5):813-6.

2. Chandrupatla TR. Quality and reliability in engineering (Vol. 2). Cambridge University Press, 2009.

3. International Organization for Standardization (ISO). Medical Laboratories-Particular requirements for quality and competence. ISO 15189:2003.

4. International Organization for Standardization (ISO). Medical Laboratories-Particular

requirements for quality and competence. ISO 15189:2012.

5. Dev Morris (2013 September 26). ISO 15189 Accreditation: Is it worth it? Retrived from http://myraqa.com/blog/iso_15189 _accreditation__is_it

6. Foundation for Quality (India). (2014).FQI ISO 15189:2012 IA and QMS Certificate course. N.P:n.p.

7. International Organization for Standardization. (2015). What is a standard? Retrieved from http://www.iso.org/iso/home/stand ards.htm 